\title{
Comparison of Ureterovesical Reimplantation Lich-Gregoir and De La Cruz-Jean Modified Techniques in Urologic Complications Post Pediatric Renal Transplantation
}

\author{
Pierre Jean Aurelus ${ }^{1 *}$, Hermilo De La Cruz Yáñez ${ }^{1}$, Amilcar Almonte Pineda ${ }^{1}$, Rafael Roberto Zapata \\ Carrión ${ }^{2}$, Ramón Eduardo Salgado Sangri' ${ }^{2}$, Marco Antonio Soto Davila ${ }^{3}$, Mario Enrique Rendón Macías ${ }^{3}$ \\ and José Alfonso Yamamoto Nagano ${ }^{1}$
}

${ }^{1}$ Department of Gastro-transplantation, Pediatric Hospital of CMNSXXI (IMSS), México

${ }^{2}$ Department of Pediatric Surgery and Urology, Pediatric Hospital of CMNSXXI (IMSS), México

${ }^{3}$ Department of Investigation, Pediatric Hospital of CMNSXXI (IMSS), México

\begin{abstract}
Introduction: Pediatric renal transplant has been more prone to complication related to technical issues at surgery. Indeed, urologic Complications are occurred in the first year of transplant. The aim of this work was to compare the rate of urologic complications with the use of two reimplantation techniques in Pediatric renal transplant.

Patients and Methods: Observational, retrospective, comparative and longitudinal cohort study based on the data from the registries of 61 renal transplants underwent modified technique (group1) and Lich-Gregoir technique (group 2) in the vesicoureteral reconstruction between January 2005 and December 2015. Categorical data were presented as frequencies and percentages and compared using Chi-square and $t$ tests.

Results: Gross hematuria was observed in $4.5 \%$ of the group 1 and $7.7 \%$ of the group 2 with $p$ value of 0.769 . None vesicoureteral reflux of the group 1 was observed, even though, $10.3 \%$ with $\mathrm{Cl} ; 0.8-19.8$ of the group 2 with a $\mathrm{p}$ value of 0.28 . Vesicoureteral stenosis of the group 1 was $4.5 \%$ and of the group 2 it was $2.6 \%$ with a p value of 1.00 .
\end{abstract}

Conclusion: Vesicoureteral reflux was more frequent in the group2 with a lower frequency of vesicoureteral stenosis than the group1.

\section{Keywords}

Ureterovesical reimplantation, Pediatric renal transplantation, Urologic complications

\section{Abbreviations}

LG: Lich-Gregoir; ESRD: End Stage Renal Disease; CMNSXXI/IMSS: National Medical Center of XXI Century of Mexican Institute of Social Security; IMSS: Instituto Mexicano Del Seguro Social

\section{Introduction}

Renal transplantation is the most effective treatment for end stage renal disease (ESRD) in whatever of both populations, adult or pediatric patients. It is considered the gold standard therapy with a better quality of life than dialyzed treatment [1-3]. Even though, renal transplantation has a standardized technique with a high rate of success, transplant in pediatric population has been more prone to complication related to technical issues at surgery [4]. Furthermore, the patients outcome are depended of multiple factors included vascular anastomosis, urinary tract reconstruction and many complications such as vascular thrombosis; leakage urinary that presented an early postoperative complication; which can significantly increase the morbidity of renal transplantation. Common etiologies are distal ureteric ischemia or technical defect. Lymphocele that is the lymph collection in

*Corresponding author: Pierre Jean Aurelus, Hospital de Pediatría Centro Médico Nacional Siglo XXI (Instituto Mexicano Del seguro Social "IMSS"), México, Tel: +525556276900

Accepted: May 12, 2020

Published online: May 14, 2020

Citation: Aurelus PJ, Yáñez HDLC, Pineda AA, et al. (2020) Comparison of Ureterovesical Reimplantation Lich-Gregoir and De La Cruz-Jean Modified Techniques in Urologic Complications Post Pediatric Renal Transplantation. J Transplant Surg 3(1):48-53 
Citation: Aurelus PJ, Yáñez HDLC, Pineda AA, et al. (2020) Comparison of Ureterovesical Reimplantation Lich-Gregoir and De La Cruz-Jean Modified Techniques in Urologic Complications Post Pediatric Renal Transplantation. J Transplant Surg 3(1):48-53

perigraft location after transplant and, it may present at any time from 2 weeks to 6 months after transplant. Retention of urine, decreased urine output, limb edema, wound dehiscence, graft loss or voiding with cicatrizal defect of the ureter junction with the bladder and vesicoureteral reflux (VUR) in the renal transplant recipient had an incident from $2 \%$ to $86 \%$. The VUR is the retrograde flow of urine from the bladder into the ureter and the renal collecting system; diagnosis is generally rendered with a voiding cystourethrogram as a result of an evaluation of a febrile urinary tract infection (UTI) or proteinuria $[3,5,6]$. Hence, The urinary tract reconstruction is performed with many types of ureterovesical reimplantation techniques, which were questioned about the most appropriate in pediatric renal recipients with a lower rate of urinary tract complications [2,4]. Indeed, reports are limited regarding ureterovesical reimplantation follow-up after pediatric renal transplantation.

The urologic complications in renal transplantation are known as the postoperative morbidity and injuries of the ureter and its junction with the bladder. They were classified as acute complications when there are presented within the first 30 days after renal transplant and late complications when there are occurred beyond 30 days of renal transplant $[1,2,7]$. Indeed, many urologic complications as well as reject, recurrent urinary tract infection and reflux are occurred in the first year of renal transplant $[4,5]$. However, vessels and urinary tract reconstructions are the most important surgical steps of the renal transplant procedure and they are associated with early loss of graft by thrombosis, leaked urinary; ureteral stenosis that can present as painless, asymptomatic hydronephrosis with rising serum creatinine and oliguria. The incidence of ureteral stenosis ranges from $2 \%$ to $7.5 \%$; hematuria and vesicoureteral reflux. Regarding the last complication, it is presented in $30 \%$ of renal transplant recipients [5-7]. Furthermore, few reports considered that vesicoureteral reflux is the harmful complication for kidney graft [4]. The aim of this work was to compare the rate of urologic complications with the use of two techniques, modified technique by De La Cruz/ Jean and Lich-Gregoir (LG), in pediatric renal transplant in a pediatric center.

\section{Patients and Methods}

\section{Subjects}

Data for this retrospective study came from the patients' registry and, the database contains patient-level, clinical outcomes, biochemical and imaging studies. All patients received urological examination with Ultrasound and cystourethrogram prior the transplant and in special case urodynamic studies were performed. The purpose of this study was to investigate the frequency of urologic complications with the use of two different techniques in vesicoureteral reimplantation in Pediatric renal transplantation, "the Modified and the LG techniques" between January 2005 and December 2015. The primary hypothesis of this study was that patient outcomes underwent Lich-Gregoir reimplantation presented equal or higher rate of urologic complications than De La Cruz-Jean modified reimplantation technique in the first year of renal transplant. The inclusion criteria were patient with aged less than 17 years with renal transplant between January 2005 and December 2015, and patients with one of those ureterovesical reimplantation techniques for the reconstruction of the urinary tract in renal transplantation.

The primary outcomes of interest in this work included overall graft survival for one year or more with registries that contain the follow-up of graft Doppler ultrasound every day within the first week of transplant, graft scan, biochemical laboratory weekly in the first month and monthly in the first year of transplant. Clinical suspects of vesicoureteral reflux were evaluated with miccional cistography. The exclusion criteria's were patients with incomplete registry and patients with anomalies of congenital defects, hip dysplasia, and reflux to the native kidney or uropathic patient. The immunosupression protocol consisted of tacrolimus or cyclosporine in combination with mycophenolate and corticosteroids. Immunologically high-risk recipients received thymoglobulin induction surgical techniques. Patients underwent surgery of renal transplantation with Lich-Gregoir reimplantation technique that consisted of an anastomosis of the renal donor ureter to the bladder mucosa of the patient recipient, which it was then buried in a muscular tunnel and intended to provide a valve effect.

On the other hand, the second technique that previously was noted by Ricard in traumatic patients and modified by De La Cruz-Jean in ureterovesical reimplantation of renal recipients. Since 2005, this modified technique is used in pediatric renal transplant performed by those authors. In brief, this technique consisted of an extraureteral reimplantation with a longitudinal incision of $1.5 \mathrm{~cm}$ that was performed at the distal ureter of the donor and this part of the ureter was inverted onto itself to create a nipple valve (Figure 1). The latter was fixed with PDS 6-O suture in both sides with inserting a double-J stent. Since, an incision is made in the bladder wall with the performing of a musculature tunnel, in which the nipple valve was fixed and the distal ureter was buried in the muscular tunnel like the LG technique. Subsequently, this vesicoureteral technique provides two antireflux mechanisms and the double-J stent was removed between 8 and 12 weeks postoperative [4,8] (Figure 2).

\section{Follow-up observations}

Monitoring began from time of the procedure until the end of the cohort observation period December 2015. There were reviewed 91 registries of patients that were divided in two groups; modified technique for group 1 and Lich-Gregoir technique for group 2. In both groups, 30 registries were excluded in the population total owing to congenital defects of the urinary tract and incomplete registries. Then, the final study populations for both ureterovesical reimplantation techniques were 61 patients, of them; group 1 had 22 (36\%) patients and 39 (64\%) patients of the group 2. The main exposure variables included recipient outcomes from living and deceased donor kidney, primary diagnosis of ESRD, recipient age at time of transplant, gender, technique of ureterovesical reimplantation, urinary leakage. Hematuria, the most typical presentation is red blood cell color visible in the urine. The hematuria is classified in gross hematuria or macroscopic he- 
Citation: Aurelus PJ, Yáñez HDLC, Pineda AA, et al. (2020) Comparison of Ureterovesical Reimplantation Lich-Gregoir and De La Cruz-Jean Modified Techniques in Urologic Complications Post Pediatric Renal Transplantation. J Transplant Surg 3(1):48-53
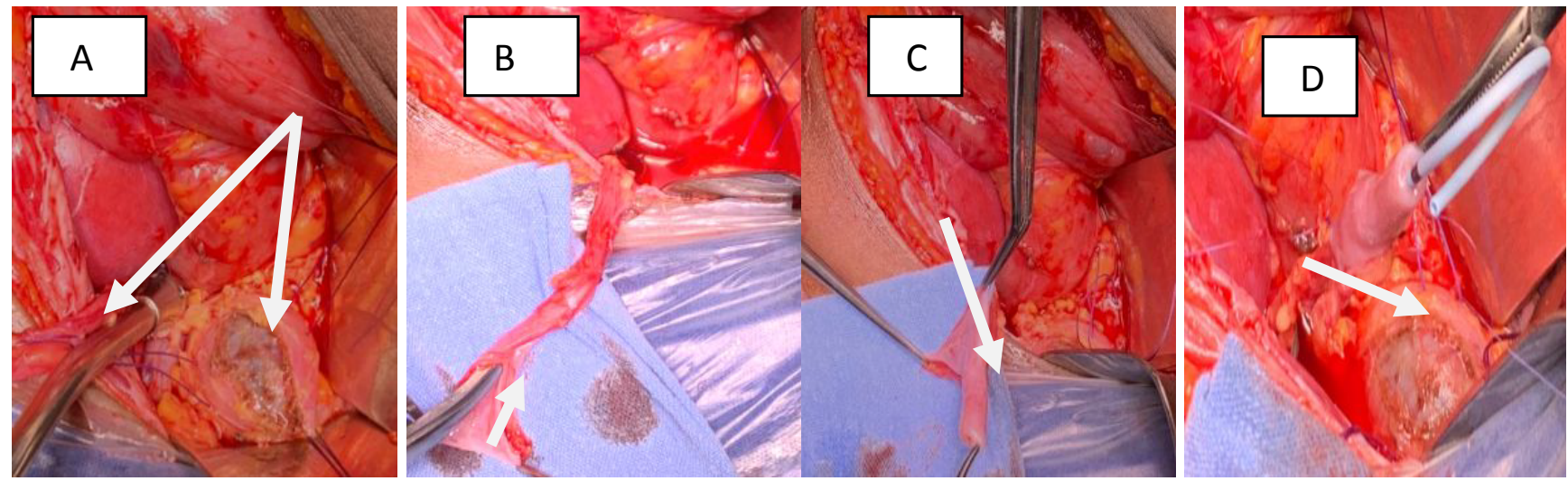

Figures 1: A: Submucosa tunnel performed at the detrusor muscular bladder after graft reperfusion and ureter exposition; B: Showing longitudinal $1.5 \mathrm{~cm}$ curt at the ureter distal; C: Nipple valve performed in the distal part of the ureter; D: Double-J inserted at the ureter.
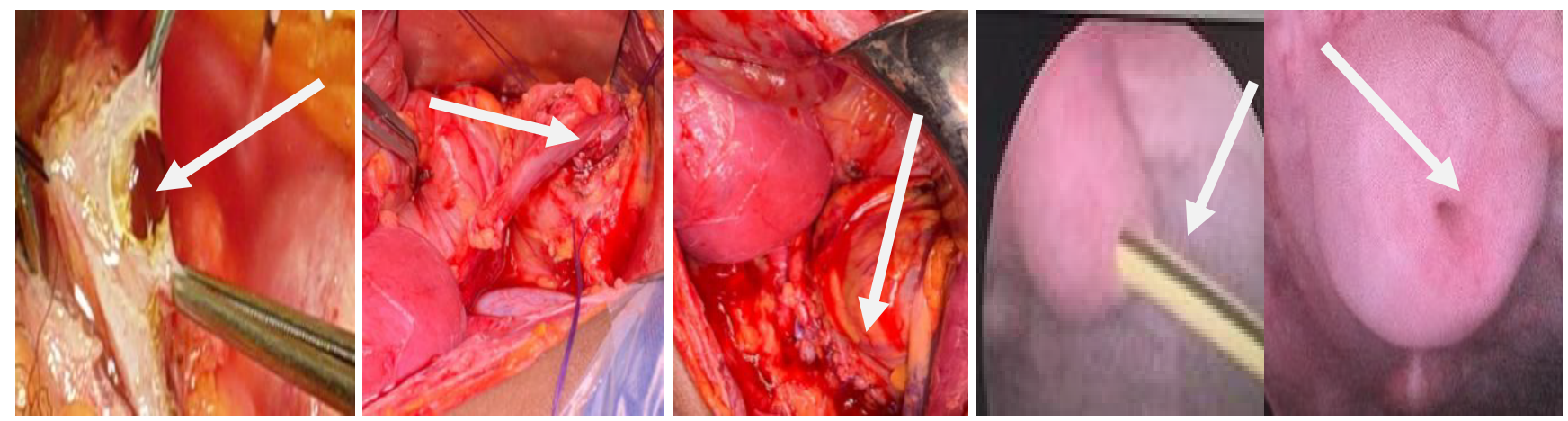

Figures 2: A: Opening bladder mucosa of $1 \mathrm{~cm}$; B: Nipple valve reimplantation; C: Locking the submucosa tunnel; D: Underwent cystoscopy procedure, image of the nipple valve with the double-J stent; E: Nipple valve with double-J removed.

maturia and microscopic hematuria when more than five reds cells or invisible color in the urine. Other hand, urinary tract infection (UTI) that is the most common infectious complication after renal transplant, it is classified into one of the four following categories: (1) Asymptomatic bacteriuria; (2) Lower UTI; (3) Acute graft pyelonephritis and (4) Urosepsis. Others important exposure variables are ureterovesical reflux to the graft, ureterovesical stenosis, acute rejection and graft loss.

\section{Statistical analysis}

This is an observational, retrospective, comparative and longitudinal cohort study based on the data from the registries of renal transplant recipients between January 2005 and December 2015. Categorical data were presented as frequencies, percentages and compared using Chi-square and t tests. Continuous variables were presented as mean with standard deviations. Analyses were performed using SPSS software version 24 and statistical significance was identified by a $p$ value of $<0.05$ and all confidence intervals used a $95 \%$ threshold. The study was approved by the local ethic committee of the hospital with the registry of R-2018-3603-042 of the Medical Center.

\section{Results}

After screening, 61 eligible patients were studied and divided in two groups, the baseline characteristics of this cohort study are shown in Table 1 . There was a preponderance of female patients in both groups ( $>60 \%)$. The mean age of patients of the group 1 is two years younger ( 7 to $9.5, \mathrm{P}<0.05$ ) than the group 2 . The primary renal diseases observed of this study were $36.4 \%$ for structural anomalies, $27.3 \%$ for glomerulonephritis of the group 1 . Therefore, the group 2 had $28.2 \%$ patients with uropathic diseases and $38.5 \%$ patients with glomerulonephritis diseases. Living and cadaveric renal transplants were performed respectively in 20 and two patients of the group 1 and there were 21 living renal transplants and 18 cadaveric renal transplants of the group 2 (Table 1 ).

The postoperative complications observed during the follow-up of this study are shown in Table 2 and there were divided in early and lastly complications in both groups. As early complications, Leakage urinary was presented in $9.1 \%$ with $\mathrm{Cl} ; 0-21.2$ of the group 1 and $7.7 \%$ with $\mathrm{Cl} ; 0-16$ of the group 2 with $\mathrm{p}$ value of 1.0; gross hematuria was observed in $4.5 \%$ with $\mathrm{Cl} ; 0-13.6$ of the group 1 and $7.7 \%$ with IC; $0-16$ of the group 2 with $p$ value of 0.769 . As lastly complications, the report presented none vesicoureteral reflux of the group 1 by contrast of $10.3 \%$ with $\mathrm{Cl} ; 0.8-19.8$ of the group 2 with a $p$ value of 0.28 ; vesicoureteral stenosis of the group 1 was $4.5 \%$ with $\mathrm{Cl} ; 0-13.6$ and of the group 2 it was $2.6 \%$ with $\mathrm{Cl} ; 0-7.6$ with a $p$ value of 1.00; none patient presented lymphocele of the group 1 and of the group 2 there were $2.6 \%$ patients with $\mathrm{Cl} ; 0-7.6$ with $\mathrm{p}$ value of 1.00 . The frequency of urinary tract 
Citation: Aurelus PJ, Yáñez HDLC, Pineda AA, et al. (2020) Comparison of Ureterovesical Reimplantation Lich-Gregoir and De La Cruz-Jean Modified Techniques in Urologic Complications Post Pediatric Renal Transplantation. J Transplant Surg 3(1):48-53

Table 1: Demographic and ureterovesical reimplantation characteristics in pediatric renal transplantation $(N=61)$.

\begin{tabular}{|c|c|c|c|c|c|}
\hline Datas & $\begin{array}{l}\text { Grc } \\
N=\end{array}$ & & & & Value of $p$ \\
\hline $\begin{array}{l}\text { Female } \\
\text { Male }\end{array}$ & $\begin{array}{l}15 \\
7\end{array}$ & $\begin{array}{l}(68.2) \\
(31.8)\end{array}$ & $\begin{array}{l}27 \\
12\end{array}$ & $\begin{array}{l}(69.2) \\
(30.8)\end{array}$ & $1.00^{*}$ \\
\hline $\begin{array}{l}\text { Age (year): } \\
\text { mean }\end{array}$ & 7 & (4) & 9.5 & (4) & $0.044^{* *}$ \\
\hline $\begin{array}{l}\text { Cause of ESRD: N (\%) } \\
\text { Glomerulophritis } \\
\text { Uropahic diseases } \\
\text { Vascular } \\
\text { Structural anomalies } \\
\text { Others }\end{array}$ & $\begin{array}{l}6 \\
4 \\
1 \\
8 \\
3\end{array}$ & $\begin{array}{l}(27.3) \\
(18.2) \\
(4.5) \\
(36.4) \\
(13.6)\end{array}$ & $\begin{array}{l}15 \\
11 \\
2 \\
3 \\
8\end{array}$ & $\begin{array}{l}(38.5) \\
(28.2) \\
(5.1) \\
(7.7) \\
(20.5)\end{array}$ & $0.106^{* * *}$ \\
\hline $\begin{array}{l}\text { Donor type: N (\%) } \\
\text { Living } \\
\text { Deceased }\end{array}$ & $\begin{array}{l}20 \\
2\end{array}$ & $\begin{array}{l}(90.9) \\
(9.1)\end{array}$ & $\begin{array}{l}21 \\
18\end{array}$ & $\begin{array}{l}(53.8) \\
(46.2)\end{array}$ & $0.004^{*}$ \\
\hline
\end{tabular}

Table 2: Urologic complications after kidney transplant underwent the type of vesicoureteral reimplantation $(\mathrm{N}=61)$.

\begin{tabular}{|c|c|c|c|}
\hline Datas & $\begin{array}{l}\text { Group } 1 \\
N=22\end{array}$ & $\begin{array}{l}\text { Group } 2 \\
\mathbf{N}=39\end{array}$ & Value of $p$ \\
\hline Early complications & $\mathrm{N}[\% ; 95 \% \mathrm{Cl}]$ & $\mathrm{N}[\% ; 95 \% \mathrm{Cl}]$ & \\
\hline -Urine leakage & $2[9.1 ; 0-21.2]$ & $3[7.7 ; 0-16]$ & $1.0^{*}$ \\
\hline $\begin{array}{l}\text {-Hematuria } \\
\text { Gross } \\
\text { Microscopic } \\
\text { Not }\end{array}$ & $\begin{array}{l}1[4.5 ; 0-13.6] \\
6[27.3 ; 6.7-45.8] \\
15[68.2 ; 48.7-87.6]\end{array}$ & $\begin{array}{l}3[7.7 ; 0-16] \\
8[20.5 ; 7.8-33.2] \\
28[71.8 ; 57.7-85.9]\end{array}$ & $0.769^{* *}$ \\
\hline \multicolumn{4}{|l|}{ Lastly complications } \\
\hline -Vesicoureteral reflux & $0[0]$ & 4 [10.3; 0.8-19.8] & $0.287^{*}$ \\
\hline -Vesicoureteral stenosis & $1[4.5 ; 0-13.6]$ & $1[2.6 ; 0-7.6]$ & $1.00^{*}$ \\
\hline -Lymphocele & $0[0]$ & $1[2.6 ; 0-7.6]$ & $1.00^{*}$ \\
\hline
\end{tabular}

infection (UTI) was $59.1 \%$ of the group 1 and $53.8 \%$ of the group 2 with one o more recurrences.

\section{Discussion}

Pediatric renal transplantation is the treatment of choice in children with ESRD. Transplantation in this population has been more prone to complication related to technical issue at surgery and strictly in the reconstruction of the urinary tract [4]. Consequently, this study retrospectively analyzed data involving 61 pediatric renal transplant cases from 2005 through 2015 and presented an original report identifying the association of postoperative urologic complications. Those urologic complications such as vesicoureteral reflux to the graft, ureterovesical stenosis, lymphocele and urine leakage among other, with the use of one of both ureterovesical reimplantation techniques (Modified Technique and Lich-Gregoir) that were analyzed at the reconstruction of the urinary tract in pediatric renal transplantation. In this regard, this report showed that the modified technique was as efficiently as the traditional Lich-Gregoir technique for ureterovesical reimplantation in the renal transplant procedure. Moreover, this modified reimplantation technique had not increased the frequency of early complications.

Hence, the primary diagnoses showed in both techniques were for the group 1: Structural anomalies such as dysplasia and polycystic kidneys in $36.4 \%$ of patients and patients for glomerulonephritis represented $27.3 \%$. While, of the group 2 the principal primary diseases were glomerulonephritis in $38.5 \%$ of patients and uropathic anomalies in $28.2 \%$ at the exception of neuropathic bladder that is a factor that may be influenced on the rate of lastly complications of the study. However, reports such as Torricelli, et al. and Turunc, et al. had similarly observations $[1,2,8]$ Living donors were the principal donors in both groups with $90.9 \%$ of the group 1 compared to $53.8 \%$ of the group 2 . Hence, the graft from cadaveric donor has major vascular and ureteral risks of com- 
Citation: Aurelus PJ, Yáñez HDLC, Pineda AA, et al. (2020) Comparison of Ureterovesical Reimplantation Lich-Gregoir and De La Cruz-Jean Modified Techniques in Urologic Complications Post Pediatric Renal Transplantation. J Transplant Surg 3(1):48-53

plications due to the cold ischemic time, vascular dissection and graft manipulation. Situations, that could be affected the group 2 with more cadaveric donors than group 1. In fact, pediatric renal transplantation has many technical challenges which are directly related with the graft quality. This tendency had seen in the Baston report in which the comparison was between Lich-Gregoir and Leadbetter-Politano ureterovesical reimplantation techniques; in this report the principal donors were also living related $[2,3,9]$.

Regarding the early complications, the rate of hematuria was major of the group 2 in this study, as well as it was in others reports. Kayler and many authors had reported hematuria in a range of 1 to $34 \%[10,11]$. The incidence of UTI after renal transplantation is very high; this study presented a trend regarding a higher incidence of UTIs of the group 2 after transplant that related with vesicoureteral reflux; the UTI is the most frequent infection after kidney transplant with a range of $35 \%$ to $79 \%$ during the first year of transplant and it is a potential risk factor for poorer graft function [7,10-15]. Lastly urologic complications: Vesicoureteral reflux was observed in $10.3 \%$ of patients with $\mathrm{Cl}: 0.8-19.8 \%$. Others complications; as ureteral stenosis with one case with $(4.5 \%, \mathrm{Cl}: 0-13.6 \%)$ and lymphocele in $2.6 \%$ with $\mathrm{Cl}: 0-7.6 \%$ of the group 2 . Furthermore, this frequency of lymphocele was observed in the first Jean reported with the use of this modified technique in pediatric renal transplantation $[4,8,16]$. Indeed, there was a preponderance of lastly urological complications in the group 2. In the Alberts report, Vesicoureteral reflux had a frequency of $12.5 \%$. While, Berli, et al. and Dinckan reported $3.8 \%$ of vesicoureteral reflux within the first sixty days of renal transplant [5,9-13]. Indeed, in this study, four patients that presented vesicoureteral reflux had congenital urologic anomalies as primary diagnosis. The frequency of vesicoureteral reflux of this work was similar to the reports of Kayler, Turunc and Torriceli with a range from $3.6 \%$ to $21.3 \%$ of the traditional technique $[2,7,8,11]$. Obstruction of urinary tract owing to ureterovesical stenosis is currently reported after renal transplantation in pediatric receiver and it is related to the bladder mucosa dissection or necrosis of the ureter distal $[5,7,11]$. On purpose, the range of this complication of this work was similarly to the literature reported, as it was observed in the report of Berli, et al. [5,6,11-21]. Only one graft was loosed owing to the humoral rejection at sixth months of transplant. Other studies reported $81.4 \%$ of survival rate of the graft at 5 years, and graft lost is not directly related to urologic complication [2,22].

\section{Conclusion}

Urinary tract reconstruction in renal transplantation is related with high rate of complications; of them, vesicoureteral reflux and ureteral obstruction owing to vesicoureteral stenosis are the most harmful to the graft. These complications are occurred in both techniques of this studied population without statistical significance. However, the population with the traditional technique had more patients with vesicoureteral reflux than patients with the modified technique; perhaps, it is for the number of recipient patients with cadaveric donors. The major limitation of this study was the lack of an important number of patients, and the modified technique was per- formed only in receiver of the living renal donors.

\section{Site}

Centro Médico Nacional Siglo XXI "Hospital de Pediatría Dr. Silvestre Frenk Freund." Department of Gastro-transplantation/Instituto Mexicano del Seguro Social (IMSS)/México.

\section{Conflicts of Interest}

There are no conflicts of interest to disclose.

\section{Acknowledgements}

To IMSS, Patient, María Del Carmen Ortega Rodríguez, Yesenia Navarro Sánchez and Jean Family.

\section{References}

1. Ali-Asgari Majid, Dadkhan Farid, Ghadian Alireza, et al. (2013) Impact of ureteral length on urological complications and patient survival after kidney transplantation. Nephro-urology monthly 5: 878-883.

2. Torricelli FCM, Watanabe A, Piovesan AC, et al. (2015) Urological complications, vesicoureteral reflux, and long term graft survival rate after pediatric kidney transplantation. Pediatric Transplantation 19: 844-848.

3. Baston C, Harza M, Preda A, et al. (2014) Comparative urologic complications of ureteroneocystostomy in kidney transplantation: Transvesical Leadbetter-Politano versus Extravesical LichGregoir Technique. Transplantation Proceeding 46: 176-179.

4. Aurelus PJ, Ortiz RC, Rendon-Macias ME, et al. (2016) Tratamiento quirúrgico de linfocele en trasplante renal pediátrico. Rev. Med Inst Mex Seguro Soc 54: 142-145.

5. Sabnis B Ravindra, Singh G abhishek, Ganpule P Arvind, et al. (2016) The development and current status of minimally invasive surgery to manage urological complications after renal transplantation. Indian J Urol 32: 186-191.

6. Berli J, Montgomery J, Segev D, et al. (2015) Surgical management of early and late ureteral complications after renal transplantation: Techniques and outcomes. Clinical Transplantation 29: 26-33.

7. Weiss A Dana, Shukla R Aseem (2015) The robotic-assisted ureteral reimplantation the evolution to a new standard. Urol Clin N Am 42: 99-109.

8. Jean A, De La Cruz H, Martínez F, et al. (2017) Modified technique by de la Cruz and Jean in pediatric kidney transplantation for ureterovesical implantation. J Urol Res 4: 1088.

9. Turunc V, Eroglu B, Tabandeh B, et al. (2017) Comparison of surgical correction techniques for post-renal transplantation vesicoureteral reflux. Transplant Proc 49: 512-516.

10. Routh J, Yu R, Kozinn S, et al. (2013) Urological complications and vesicoureteral reflux following pediatric kidney transplantation. The Journal of Urology 189: 1071-1076.

11. Dinckan Ayhan, ALiosmanoglu Ibrahim, Kocak Huseyin, et al. (2013) Surgical correction of vesico-ureteric reflux for recurrent febrile urinary tract infections after kidney transplantation. BJU International 112: E366-E371.

12. Kayler L, Kang D, Molmenti E, et al. (2010) Kidney transplant ureteroneocystostomy techniques and complications: Review of the literature. Transplant Proc 42: 1413-1420. 
Citation: Aurelus PJ, Yáñez HDLC, Pineda AA, et al. (2020) Comparison of Ureterovesical Reimplantation Lich-Gregoir and De La Cruz-Jean Modified Techniques in Urologic Complications Post Pediatric Renal Transplantation. J Transplant Surg 3(1):48-53

13. Alberts VP, Minnee RC, Bemelman FJ, et al. (2012) Ureteral reconstruction after renal transplantation: Clinical outcome and risk factors. Urol Int 88: 333-337.

14. Camargo LF, Esteves ABA, Ulisses LRS, et al. (2014) Urinary tract infection in renal transplant recipient: incidence, risk factors, and impact on graft function. Transplant Proc 46: 1757-1759.

15. Golebiewska E Justyna, Slizien-Debska Alicja, Rutkwowski Boleslaw (2014) Urinary tract infections during the first year after renal transplantation: one center's experience and a review of the literature. Clin Transplant 28: 1263-1270.

16. Ahmed A Shokeir, Yasser Osman, Bedeir Ali-El-Dein, et al. (2005) Surgical complications in live-donor pediatric and adolescent renal transplantation: Study of risk factors. Pediatr Transplantation 9: 33-38.

17. Mahdavi Zafarghandi, Afarghandi R, Sheikhi Zh (2013) Extensive ureteral stricture in renal transplant recipients: Prevalence and Impact on graft and patient survival. Int J Organ Transplant Med 4: 165-171.
18. Bagga Herman, Lin S, Williams A, et al. (2016) Trends in renal transplantation rates in patients with congenital urinary tract disorders. J Urol 195: 1257-1262.

19. Penna F, Lorenzo A, Farhat W, et al. (2017) Ureteroureterostomy: An alternative to ureteroneocystostomy in select cases of pediatric renal transplantation. The Journal of Urology 197: 1-5.

20. Vasdev N, Coulthard M, Lambert H, et al. (2012) The modified Barry technique to prevent vesicoureteral reflux in paediatric renal transplant recipients: Initial recipient outcomes. J Pediatr Urol 8: 97-102.

21. Lee S, Moon T-S, Kim Y. Roh, et al. (2013) Presence of vesicoureteral reflux in the graft kidney does not adversely affect long-term graft outcome in kidney transplant recipients. Transplant Proc 45: 2984-2987.

22. Guzman JA (2012) Intravesical ligation as a new technique to manage a refluxing native ureter without simultaneous nephrectomy in renal transplantation. Transplant Proc 44: 2940-2944. 